\title{
Aux origines du Directoire : le « proconsulat » de Jacques Reverchon (brumaire-ventôse an IV)
}

\section{Bernard Gainot}

\section{Q OpenEdition \\ 1 Journals}

Édition électronique

URL : https://journals.openedition.org/ahrf/829

DOI : 10.4000/ahrf.829

ISSN : 1952-403X

Éditeur :

Armand Colin, Société des études robespierristes

Édition imprimée

Date de publication : 1 juin 2003

Pagination : 129-146

ISSN : 0003-4436

\section{Référence électronique}

Bernard Gainot, « Aux origines du Directoire : le « proconsulat » de Jacques Reverchon (brumaireventôse an IV) ", Annales historiques de la Révolution française [En ligne], 332 | avril-juin 2003, mis en ligne le 22 avril 2008, consulté le 24 avril 2022. URL : http://journals.openedition.org/ahrf/829 ; DOI : https://doi.org/10.4000/ahrf.829 


\title{
AUX ORIGINES DU DIRECTOIRE : LE «PROCONSULAT » DE JACQUES REVERCHON (BRUMAIRE-VENTÔSE AN IV)
}

\author{
BERNARD GAINOT
}

\begin{abstract}
Les derniers représentants envoyés en mission par la Convention nationale reçoivent leurs instructions dans un contexte très spécifique, celui des lendemains de la journée'parisienne du 13 vendémiaire an IV. C'est la raison pour laquelle nous parlons de « missions vendémiaristes ».

Ces représentants sont peu nombreux; le plus connu est Louis-Stanislas Fréron, envoyé dans les départements autour de Marseille. Nous nous arrêtons ici sur Jacques Reverchon, qui exerça une mission absolument identique dans la grande région lyonnaise.

Leurs pouvoirs sont considérables ; épuration des autorités nouvellement élues, réintégration dans l'appareil administratif du personnel révolutionnaire désarmé au printemps, remise en vigueur des lois d'exception contre les émigrés et les prêtres réfractaires.

Ces pouvoirs sont fatalement ambigus dans une période de transition entre le gouvernement révolutionnaire et le gouvernement constitutionnel ; prolongement du régime de salut public, ils traduisent pourtant le rééquilibrage des centres de décision en faveur de l'Exécutif. La justification ultime de la survie du régime révolutionnaire est donnée par l'existence d'une " grande conspiration » royaliste aux ramifications nationales (les Compagnons de Jésus, les Compagnons du Soleil).

Ce faisant, ces missions témoignent aussi de l'impossibilité de terminer la Révolution, et de faire appliquer une authentique loi d'amnistie.
\end{abstract}

Mots clés : commissaires du pouvoir exécutif ; représentants en mission ; régime révolutionnaire ; élections; amnistie; épuration; ordre public ; Compagnons de Jésus ; Compagnons du Soleil.

À partir du 20 vendémiaire an IV (12 octobre 1795), se tiennent les assemblées électorales pour choisir les premiers représentants au Corps législatif du Directoire, mais aussi les nouveaux administrateurs départementaux. À Mâcon, en Saône-et-Loire, ces nouveaux administrateurs sont élus le 27 vendémiaire (19 octobre), et désignent immédiatement un 
commissaire pour remplir les fonctions provisoires d'agent du Pouvoir exécutif, prévues par la nouvelle Constitution ; c'est Lejeune Desmaisons.

Or, le même jour, arrive Jacques Reverchon, envoyé en mission par le Comité de sûreté générale dans les départements du Rhône, de la Loire, de l'Ain, de la Saône-et-Loire, et de l'Isère. Le commandant de la Garde nationale de Mâcon vient à 19 heures prévenir les électeurs que nombre de citoyens armés se sont introduits dans la Maison commune. Ces citoyens ont été appelés par la municipalité de Mâcon pour fournir une garde d'honneur au commissaire Reverchon.

Le lendemain, un nouveau rassemblement armé se tient à la Maison commune, en présence de Reverchon et des autorités municipales, mais sans la participation des chefs de la Garde nationale. Ce qui provoque, le 29 vendémiaire, la suspension de la municipalité par la nouvelle administration départementale.

Deux administrateurs du département se rendent chez Reverchon, pour lui demander de présenter ses pouvoirs. Ce dernier les reçoit sèchement, rétorque qu'il attend la fin des opérations électorales. Puis il fait parvenir deux arrêtés, dont l'un destitue le commandant de la Garde nationale. Le commissaire Lejeune Desmaisons refuse d'enregistrer ces arrêtés, arguant que la loi du 18 prairial an III réserve à l'élection le choix des postes de commandement dans la Garde nationale sédentaire. À 21 heures, un détachement armé vient arrêter le commissaire.

Le lendemain, Reverchon se présente à l'administration centrale. Il justifie l'arrestation de Lejeune et son transfert à Paris par les lois des 3 brumaire (émigration) et 14 vendémiaire (coalition contre la sûreté de l'État, en raison d'une participation aux manœuvres contre-révolutionnaires des sections parisiennes) de l'an IV. Il présente ses pouvoirs comme représentant du peuple commissaire provisoire du gouvernement désigné par le Comité de sûreté générale. Il confirme la suspension de trois administrateurs, prononcée par le Comité de législation de la Convention, pour «coalition » avec le commissaire Lejeune Desmaisons. Puis il nomme de nouveaux administrateurs et un nouveau commissaire (1).

Le 15 frimaire (6 décembre 1795), le Directoire confirme ces suspensions et nominations. Ce qui autorise Reverchon à intervenir de la même façon dans les autres départements placés sous son autorité. Le 29 vendémiaire, il avait également suspendu un administrateur du département de l'Ain. Cette suspension n'avait pas eu de suites, Reverchon n'ayant pas le don d'ubiquité. Il intervient là où il est physiquement présent, mais aussi là où il peut contrôler l'exécution en s'appuyant sur la force armée. À 
Bourg, cette dernière n'est pas sûre. Or, Reverchon attend le moment propice au renversement de la situation : le 26 frimaire an IV (17 décembre), il signe à Mâcon un arrêté qui ordonne au chef de la brigade du $20^{\mathrm{e}}$ régiment de dragons stationné à Bourg, Gondran, de prendre le commandement de cette place stratégique pour l'approvisionnement de l'Armée des Alpes.

Le lendemain, 27 frimaire, le détachement de dragons cerne le bâtiment de l'administration centrale à Bourg, et effectue des perquisitions dans les locaux, pour rechercher des individus à arrêter.

Le commandant de la place est convoqué pour rendre compte à l'administration centrale. Il assure qu'il est en possession d'un mandat d'arrêt signé «Reverchon», visant plusieurs personnes qui doivent être transférées à Mâcon. L'administration centrale délègue alors l'un de ses membres auprès de Reverchon, qui est toujours désigné comme le «représentant du peuple actuellement à Mâcon ». Même accueil froid et distant que pour les délégués des administrateurs de Saône-et-Loire, le 30 frimaire (21 décembre). Il se contente de transmettre à Bourg un arrêté, toujours signé simplement du « représentant du peuple Reverchon».

«Instruit que les ganses blanches que portent les chasseurs de la Garde nationale sédentaire de Bourg, chef-lieu du département de l'Ain, ont été la cause des mouvements qui se manifestent dans ce département. Considérant qu'il est instant de faire disparaître tout signe de ralliement dont la malveillance pourrait faire usage, que rappeler le Français à servir la Patrie sous le même uniforme, c'est seconder les vœux du gouvernement qui redouble d'efforts pour faire de la Nation entière une seule famille.

$\mathrm{Vu}$ la loi qui a licencié la compagnie des grenadiers et des chasseurs de Paris, les procès-verbaux et les pièces relatifs aux mouvements qui ont agité la commune de Bourg dans le courant de frimaire [...]

Arrête ce qui suit :

Article $1^{\text {er }}$ : les compagnies de grenadiers et de chasseurs de la Garde nationale sédentaire du département de l'Ain sont licenciées [...]

Article 2 : Ils rentreront dans les compagnies du Centre, où ils seront de service commun aux citoyens soldats qui les composent. » (2)

L'administrateur suspendu, Rousset, juge plus prudent de démissionner le 3 nivôse. L'administration centrale lui choisit un successeur. Mais Reverchon a son propre candidat, Lecœur. Ce dernier se présente pour occuper son poste le 6 nivôse. Comme sa nomination continue d'être contestée, Reverchon se déplace en personne devant l'administration centrale le 10 nivôse.

(2) A.D. Ain, L 114, Registre de délibérations de l'administration centrale, arrêté du 30 frimaire an IV, enregistré à Bourg le $1^{\mathrm{er}}$ nivôse. 
Il justifie enfin la destitution de Rousset par son implication dans les massacres des anciens membres du comité révolutionnaire de Bourg (3) : s'il ne fut pas un participant actif, du moins, comme procureur-syndic du district, a-t-il « autorisé par un coupable silence le cours odieux des assassinats ».

Il présente ensuite un arrêté de nomination d'un nouveau commissaire départemental pour l'Ain, en même temps que celui de la nomination de Lecœur. Les administrateurs protestent, rétorquent qu'il faut s'appuyer sur « la charte de nos droits et de nos devoirs », notamment sur l'article 188, qui attribue exclusivement aux administrations départementales et municipales le droit de s'adjoindre temporairement un administrateur pour exercer en cette qualité, jusques aux élections suivantes, lorsqu'elles perdent un ou plusieurs de leurs membres, par mort, démission, ou autrement.

«La loi du 29 vendémiaire an IV attribue certes aux représentants du peuple en mission le droit de destituer les fonctionnaires publics; mais elle ne s'explique pas sur leur mode de remplacement... »

Finalement, un compromis s'ébauche. Le candidat de l'administration renonce, et Reverchon cesse de son côté de vouloir imposer Lecœur.

Des interventions, sur lesquelles nous reviendrons, ont également lieu dans la Nièvre, dans la Loire...

L'historiographie n'a guère accordé d'attention à ces épisodes absolument cruciaux pour l'installation du régime constitutionnel, ou plus précisément pour la transition du régime révolutionnaire au régime constitutionnel (4). Les historiens focalisent l'analyse sur les débats au sein de la Convention, et sur le nouveau rapport de forces qui s'affirme entre la journée du 13 vendémiaire an IV et la loi dite d'amnistie du 3 brumaire an IV. Expression de ce nouveau rapport de forces, la mission «vendémiariste » (5) de Fréron dans les départements du Midi est mise en exergue; or, cette mission si particulière est en tous points analogue à celle de Jacques Reverchon (6).

(3)Ibid., L 115; Jérôme CROYET, La mission du représentant Albitte dans l'Ain, 28 nivôse-18 floréal an II, mémoire de maîtrise sous la direction de S. Chassagne, Université Louis Lumière Lyon II, 1996.

(4) Ludovic SCIOUT, Le Directoire, Paris, 1895, tome 1 ; Georges LEFEBVRE, La France du Directoire, avant-propos et notes de J.-R. Suratteau, Paris, Messidor-Éditions sociales, 1983 (réédit.); Denis WORONOFF, «La République bourgeoise », dans Nouvelle histoire de la France contemporaine, tome 3, Paris, Le Seuil, 1972.

(5) Terme que nous proposons d'employer désormais à la place de «mission tardive », pour les raisons que nous exposons plus loin.

(6) Louis-Stanislas FRÉRON, Mémoire historique sur la réaction royale et sur les massacres du Midi, avec des notes et des pièces justificatives, par le citoyen Fréron..., Paris, Lyon, Marseille, an IV; Paul GAFFAREL, «Le second proconsulat de Fréron à Marseille », La Révolution française, 1916, pp. 148-149 et 313-336. L'auteur se contente toutefois de recenser les interventions du commissaire, sans préciser la nature des pouvoirs dont il a été investi, et le contexte de cette dévolution. Ainsi, cette mission spéciale est ramenée à une simple mission de « pacification » thermidorienne. 
Les questions posées sont de plusieurs ordres : toutes ne seront pas évoquées dans ce texte. En premier lieu, il faut analyser l'articulation entre les pouvoirs locaux et les véritables centres parisiens de décision (centralité législative et acteurs politiques). Ensuite, il y a nécessité de fortement contextualiser : quel est le rapport entre ce «moment» (7) particulier de vendémiaire-frimaire an IV, et la structure institutionnelle, l'agencement interne des pouvoirs dans la République constitutionnelle? Où est la légitimité? Dans les élections, affirment avec force les nouveaux administrateurs :

«Si ce n'est pas un droit illusoire accordé au Peuple d'élire ses magistrats, ce ne peut donc pas être sans motifs bien puissants et bien légitimes qu'il est permis de porter atteinte à son choix. » (8)

Et les mêmes répandent la version du «proconsulat» de Reverchon, tout à la fois régime de l'arbitraire et protectorat des terroristes. Le représentant :

«idole de nos exclusifs qu'il soutient avec un dévouement opiniâtre [...] Il arrive en vendémiaire an IV, suspend les cinq élus du peuple à l'administration centrale [...] il fait traduire à Paris le commissaire provisoire, nommé par l'administration légitime, comme suspect d'avoir pris une part active aux événements de vendémiaire ; et suspend les administrateurs, comme soupçonnés d'avoir trempé dans les mêmes mesures liberticides, sous le prétexte qu'ils ont nommé ce commissaire provisoire. » (9)

Ce à quoi les autorités centrales et leurs représentants répliquent au nom d'une supériorité du Législateur sur le citoyen préalablement égaré dans ses choix par des passions et des pressions morales perverses :

«La réaction du 9 thermidor avait fait appeler aux places les plus importantes des hommes ennemis de la République, et totalement vendus au parti royaliste : ces hommes voient avec peine s'organiser le gouvernement républicain, et ils forment une espèce de coalition pour entraver dans leur marche les autorités populaires. Les choix des assemblées électorales ont été mauvais, surtout pour les administrations départementales; par contrecoup, se trouve mauvais le choix des commissaires nationaux provisoires près les différents cantons. » (10)

(7) Françoise BRUNEL et Jacques GUILHAUMOU, «Les moments de la Révolution française et la synthèse politique (1789-1795) », dans Recherches sur la Révolution, Paris, La Découverte, 1991, pp. 45-54.

(8) A.D. Isère, Papiers Reverchon, $1 \mathrm{~J} 380$ : réclamation au nom des administrateurs du département de Sâne-et-Loire au pouvoir exécutif, sur la suspension provisoire de leurs administrations, 26 nivôse an IV (16 janvier 1796). (avril 1797)

(9) A.N., FlcIII, Saône-et-Loire 12, brochure sur les troubles de Mâcon en germinal an V

(10) Ibid, AFIII 32, Papiers du ministre de la Justice Merlin de Douai État des pièces relatives à la mission du citoyen Bezout dans le département de la Nièvre, 21 brumaire an IV - 20 novembre 1795. (Déclaration des administrateurs municipaux de Moulins). 
La querelle de légitimité renvoie bien évidemment à la nature des pouvoirs de ces envoyés : représentants du peuple? Mais alors ils sont un nouvel avatar des représentants en mission de la Convention nationale. $\mathrm{Ce}$ sont les missions tardives, avec tout ce que ce terme peut véhiculer de crépusculaire, et non d'inaugural. Commissaires du gouvernement? En passant du côté de l'Exécutif, on passe sur le versant de l'avenir, et ces «proconsuls » anticipent le rôle des préfets extraordinaires. Ils sont parfois qualifiés de commissaires de la Convention nationale, parfois de commissaires du gouvernement (11). Redoutable ambiguïté, en contradiction avec les ambitions affichées dans la loi du 5 fructidor an III - 22 août 1795 : Terminer la Révolution.

«... Article 10 : Les membres qui, à l'époque de la formation des deux conseils, composeront les Comités de salut public et de sûreté générale, continueront provisoirement leurs fonctions jusqu'au jour de l'installation du Directoire.

Article 11 : À dater du jour de cette installation, les comités ne pourront prendre, ni signer aucun arrêté : ils fourniront au Directoire les éclaircissements dont il aura besoin.

Article 12 : Toutes les commissions exécutives continueront leurs fonctions jusqu'à ce que le Directoire ait organisé le ministère ; et tous les fonctionnaires publics jusqu'à ce qu'ils aient été renouvelés dans la forme prescrite par la Constitution. » (12)

Redoutable ambiguïté, qui se trouve dans les termes mêmes de la loi : la période transitoire, en principe, ne peut excéder sept jours. Les deux conseils législatifs ouvriront leurs séances le lendemain au plus tard de la dernière séance de la Convention nationale. Dans trois jours, pour tout délai, le Conseil des Cinq-Cents présentera au Conseil des Anciens une liste de cinquante noms pour former le Directoire exécutif. Le Conseil des Anciens choisira les cinq directeurs dans cette liste dans les trois jours qui suivront sa présentation.

Si nous comprenons bien, dans cette période transitoire, ce sont les Comités de salut public et de sûreté générale qui assument le Pouvoir exécutif, ce qui est contradictoire avec leur nature même de simples émanations du Législatif, et avec la théorie constitutionnelle de séparation des pouvoirs. Quant aux commissions exécutives censées rester en fonctions jusqu'à l'installation des divers ministères, elles ne devraient pas concerner les représentants du peuple qui ont reçu des missions des Comités de gouvernement. Ce régime transitoire est donc dérogatoire au fonctionnement du régime constitutionnel.

(11) C'est le titre le plus fréquemment attribué à Etienne Bezout.

(12) Loi sur les moyens de terminer la Révolution, 5 fructidor an III (22 août 1795). Dans Bulletin des lois $n^{\circ} 181,1102$, Collection Duvergier, t. VIII, p. 301 : De la mise en activité de la Constitution. 
Pour avaliser cette dérogation aux principes constitutionnels, il faut admettre une situation d'exception. Cette situation est créée par la révolte des sections parisiennes le 13 vendémiaire. Mais pas seulement, comme nous le verrons : les thermidoriens justifient ces moyens exceptionnels par la situation existant dans de nombreux départements (dont ceux qui font l'objet de la présente étude, mais aussi dans les départements méridionaux situés dans le rayon d'action de Fréron, dans les départements belges en instance d'annexion, dans les départements de l'Ouest soumis à l'autorité militaire), situation qui met en péril la République. Le gouvernement révolutionnaire se trouve ainsi prolongé au sein même de la nouvelle configuration des pouvoirs. La Révolution n'est pas terminée...

Qui est Jacques Reverchon? Un personnage paradoxal, qui résume bien toutes les singularités de l'époque. C'est un illustre inconnu qui a exercé de grands pouvoirs. C'est un représentant quasi inamovible de 1791 à 1799, dont l'essentiel de l'action se déroule hors de l'enceinte parlementaire, en province ou dans les coulisses du pouvoir.

Figure socialement représentative, son parcours professionnel rappelle celui de Benoît Lacombe décrit par Joël Cornette comme emblématique d'un révolutionnaire ordinaire (13). Né près de Lyon en 1750, à Saint-Cyr au Montd'Or, dans une famille de notables enrichis par le négoce, c'est tout naturellement vers cet état qu'il se tourne. Il est, dans une première partie de son existence, l'homme des réseaux commerciaux, notamment celui du commerce du vin entre Beaujolais et Mâconnais, entre production et commercialisation. Installé à Marcigny sur la Loire où il a des entrepôts, puis à Vergisson au cœur du vignoble mâconnais, il prend des participations dans toutes les entreprises de grossistes qui approvisionnent le marché parisien en vins de la région.

Il représente bien le Tiers État dans sa dimension roturière d'hommes nouveaux. Pour passer du plan social au plan politique, il faut une assise. C'est la franc-maçonnerie qui lui offre cette opportunité. L'homme nouveau devient notable mâconnais, ayant pignon sur rue entre l'hôtel de ville et les quais de la Saône. Élu à l'administration départementale en 1790, il devient législateur en 1791, conventionnel en 1792, représentant en 1795 . Il ne prend jamais, ou quasiment jamais, la parole à l'Assemblée. Engagé aux côtés de la Montagne, c'est lui qui organise la résistance au fédéralisme lyonnais, qui supervise l'approvisionnement en hommes et en armes de l'armée conventionnelle qui a établi son état-major à Limonest, que Reverchon ne quitte pas jusqu'à la victoire (14). Mâcon fait un peu figure de base

(13) Joël CORNETTE, Un révolutionnaire ordinaire, Benoit Lacombe, négociant (1759-1819), Seyssel, Champ Vallon, 1986.

(14) Edouard HERRIOT, Lyon n'est plus, tome 2, Le siège, Paris, Hachette, 1938 ; A.D. Isère, Papiers Reverchon, $1 \mathrm{~J} 374$. 
arrière de l'armée devant Lyon, tandis que le vaste rayon d'action du représentant s'étend sur les départements qui seront de sa compétence en l'an IV : l'Ain, l'Isère, la Loire. Des contacts se nouent, des réseaux locaux se tissent. Hors ces liens d'homme à homme, ces fidélités, le pouvoir d'un représentant n'est rien.

La mémoire historique a retenu sur le devant de la scène des noms plus illustres, Fouché, Collot d'Herbois, et même Dubois-Crancé, dont Reverchon est alors extrêmement proche.

Reverchon se retrouve à Lyon en l'an II, et surtout en l'an III, pour des missions «thermidoriennes». Mais d'un caractère spécial; comme DuboisCrancé, comme son collègue Poullain-Grandprey, Reverchon veut infléchir le cours de la politique conventionnelle dans le sens « républicain ». Il faut certes arrêter les massacres qui ont pris l'allure de la «terreur blanche » en mai 1795 (15), mais ne pas s'en tenir là : garantir un asile sûr, trouver des emplois à l'ancien personnel de l'an II, que Reverchon connaît bien, souvent nominalement. Trouver des emplois ne doit pas être entendu seulement au sens trivial (occuper des postes dans l'administration). Il y a toute une conception « régénératrice » de l'économie chez Reverchon, qui se traduit par le projet jacobin de fondation d'entreprises républicaines (16), exposé dans la brochure sur la réhabilitation du commerce de Commune-Affranchie (17).

Reverchon est donc bien inséré dans les réseaux du pouvoir parisien, étape ultime de sa carrière publique et privée. $\mathrm{D}$ fut longtemps membre du Comité de sûreté générale, il est proche en l'an IV des puissants du jour, Merlin de Douai, et surtout le futur directeur Reubell (18). C'est donc sans surprise que la mission vendémiariste lui est confiée. Sa bonne connaissance du terrain, ses relations locales, les éminents services rendus en 1793, puis en 1795, le désignent particulièrement. Ses adversaires ne manquent pas de souligner à quel point de tels atouts sont discutables : les administrateurs de Saône-et-Loire, lorsqu'ils réclament l'examen des pouvoirs du commissaire, précisent que recevoir de tels pouvoirs dans un département dont on est originaire est contraire à la loi (19). Ils anticipent en fait sur le régime consulaire.

(15) Renée FUOC, La réaction thermidorienne à Lyon, Lyon, Federop, 1989 ; Bruno BENOIT, «Chasser le mathevon à Lyon en l'an III », dans Le tournant de l'an III (dir. Michel VOVELLE), Paris, Éd. du CTHS, 1997, pp. 497-508.

(16) Reverchon cherchera à mettre en pratique ce projet sous le Directoire, et au cours des années suivantes, en se faisant le protecteur et principal actionnaire de la manufacture de papiers peints du réfugié lyonnais Joseph Dufour. Un centre Joseph Dufour vient de s'ouvrir à Tramayes, dans le Haut-Mâconnais : on peut y trouver de nombreux documents concernant cette manufacture.

(17) BNF, Res. Z Lb (41) 1095, Mémoire au Comité de salut public sur la réhabilitation du commerce de Commune-Affranchie., 4 prairial an II.

(18) Il y eut un projet de mariage (qui n'aboutit pas) entre le fils aîné de Reubell et la fille de Reverchon. Cf. Jean-René SURATTEAU et Alain BISCHOFF, Jean-François Reubell, l'Alsacien de la Révolution française, Strasbourg, Éditions du Rhin, 1995.

(19) A.D. Isère, Papiers Reverchon, 1J 380. 
Quels sont donc ces fameux pouvoirs?

«Le Comité de sûreté générale, en vertu de la loi du 14 vendémiaire qui l'autorise à envoyer des représentants du peuple en mission, arrête que le représentant du peuple Reverchon se rendra sur-le-champ dans les départements de Saône-et-Loire, de l'Isère, de l'Ain, de la Loire et du Rhône, pour y continuer les opérations de concert avec le représentant Poullain-Grandprey, il se conformera d'ailleurs aux instructions données par le Comité. » (20)

Remarquons que le Comité de sûreté générale est alors dominé par les thermidoriens «de gauche», front républicain des anciens Montagnards comme Collombel, Monmayou, Bordas, et Roberjot, et d'anciens Girondins comme Pierre Guyomar. Reverchon est très proche de Collombel, et surtout de Claude Roberjot, l'ancien curé de Saint-Pierre de Mâcon.

Des deux comités de gouvernement, c'est le Comité de sûreté générale qui a désormais l'ascendant sur le Comité de salut public. Déséquilibre significatif, qu'il faudrait interroger davantage, lorsqu'on sait qu'à l'époque, le Comité de salut public est dominé par les membres de la Commission des Onze qui ont rédigé l'acte constitutionnel (21).

La position particulière du Comité de Sûreté générale est sensée exprimer le régime transitoire entre la Convention et le Directoire, ses pouvoirs anticipant en quelque sorte ceux du Directoire exécutif: «Avant que le Directoire exécutif fût installé, quoique la Constitution fût imparfaitement en activité, le Comité de sûreté générale de la Convention nationale était désigné pour en tenir lieu dans la partie administrative... »(22).

Le débat à la Convention oppose ceux qui souhaitent que les pouvoirs de ces représentants cessent avec la mise en route du Directoire (Doulcet : « on ne peut prolonger sans troubles les moyens du gouvernement révolutionnaire dans l'état du gouvernement constitutionnel ») et ceux qui arguent du transfert des compétences dans le régime constitutionnel pour prolonger les pouvoirs des représentants en mission (Merlin de Douai, Génissieu : «si on distingue soigneusement le pouvoir exécutif et le pouvoir législatif, la Constitution ne peut être établie que par les résultats des choix faits par les assemblées électorales. En attendant, il faut bien que quelqu'un gouverne... ») En fin de compte, ces derniers l'emportent :

«La Convention nationale décrète que les représentants du peuple envoyés dans les départements ou aux armées qui ne seront pas rappelés à l'époque du 5 brumaire prochain, soit qu'ils aient été réélus au Corps législatif (23), ou non, continueront leur mission en qualité de commissaires du

(20) A.D. Ain, L 114. pp. 20-21.

(21) Bernard GAINOT, Dictionnaire des membres du Comité de salut public, Paris, Tallandier, 1990,

(22) AD. Isère, Papiers Reverchon, $1 \mathrm{~J} 380$.

(23) C'est le cas de Jacques Reverchon. 
gouvernement, jusqu'à ce que le Directoire exécutif leur ait donné avis de son entrée en exercice des fonctions qui lui sont attribuées par la Constitution. » (24)

Ce texte, qui porte l'empreinte de Merlin de Douai, institue les comités de gouvernement, et singulièrement le Comité de sûreté générale, en gouvernement provisoire de la République, avec des envoyés dans les départements qui ne peuvent être que munis de pouvoirs exceptionnels puisqu'ils vont destituer des autorités élues aux termes d'une Constitution supposée mettre fin au régime d'exception !

Une telle construction est marquée du sceau de l'improvisation. Une fois le Directoire installé, Merlin de Douai, ministre de la Justice, va s'attacher, avec toute la rigueur dont il sait faire preuve (25), à légaliser, non plus les pouvoirs de l'ensemble des envoyés en mission, mais leurs actions, quitte à donner des pouvoirs de l'ensemble des représentants en mission une lecture quelque peu différente de celle qui est couramment admise (le législatif ambulant [26]) :

«La loi du 20 vendémiaire ne dit pas que leur mission devait cesser au 5 brumaire. Elle dit seulement que la mission continue sous un autre nom; en fait, le nom qu'ils auraient toujours dû porter, puisque toujours les représentants du peuple en mission ont été, non les délégués du Corps législatif, mais les commissaires du gouvernement qui, alors par accident, était concentré dans le Corps législatif même. »

Les pouvoirs de la Convention, comme corps constituant ne peuvent se borner à la confection d'une Constitution : «Ils s'étendent à tous les actes propres à faire marcher son ouvrage et en assurer la durée » (27).

Par conséquent, les instructions données aux commissaires sont tout à fait légales, et ne cessent que lorsque le Directoire estime que la mission a été remplie :

«1. Surveiller les prêtres réfractaires, poursuivre et faire punir ceux d'entre eux qui prêchent la contre-révolution.

2. Faire exécuter les lois rendues contre les émigrés rentrés.

3. Faire arrêter et punir les auteurs de la conspiration contre-révolutionnaire.

4. Épurer et renouveler au besoin les autorités constituées.

Ranimer le patriotisme, faire exécuter les lois, respecter la Convention nationale. » (28)

(24) Le Moniteur Universel, séance du 20 vendémiaire an IV - 12 octobre 1795 ; Recueil des actes du Comité de salut public, séance du 20 vendémiaire an IV (12 octobre 1795), pp. 387-388.

(25) Hervé LEUWERS, Un juriste en politique, Merlin de Douai, 1754-1838, Arras, Artois Presse Université, 1996.

(26) Michel BIARD, Missionnaires de la République. Les représentants du peuple en mission (1793-1795), Paris, Éd. du CTHS, 2002. an IV.

(27) A.N., AFIII32, Papiers de Merlin de Douai; avis sur les pouvoirs du citoyen Bezout, 16 frimaire

(28) Ibid. 
Merlin va légaliser les actes des commissaires ; destitution des fonctionnaires suspects, puis nomination de nouveaux administrateurs, et surtout de nouveaux commissaires auprès des municipalités de cantons ; et libération, souvent accompagnée du réarmement, du personnel jacobin placé sous surveillance depuis le printemps. Reverchon reprend les arguments des «patriotes de 1789 », qui démontrent que les opérations de l'assemblée électorale de la Loire :

«... ont été dominées par le fanatisme et l'intrigue ; des émissaires envoyés dans les campagnes voisines ont corrompu l'esprit public, empoisonné l'opinion du peuple, en lui disant sans doute que si l'on mettait des patriotes en place, c'en était fait de la religion dominante, catholique, apostolique, et romaine, que le règne de la Terreur renaîtrait, ainsi que le renversement des autels. » (29)

Arrêtons-nous un peu sur l'action d'Etienne Bezout dans la Nièvre. Etienne Bezout (neveu du mathématicien auquel Monge a succédé dans les jurys des écoles navales) arrive à Nevers le 2 brumaire an IV - 24 octobre 1795. Le 21 brumaire (12 novembre), il convoque à son domicile les administrateurs départementaux qui viennent de prendre un arrêté pour éloigner de Nevers les amnistiés libérés par la loi du 4 brumaire. Les administrateurs rétorquent que l'administration en corps ne doit jamais se déplacer hors du lieu de ses séances, et se plaignent de ce que Bezout ait requis la force armée pour protéger les membres de l'ancien comité de surveillance de Cosne-sur-Loire. L'un des membres de cet ancien comité n'est autre que le propre secrétaire de Bezout.

Le 22 brumaire (13 novembre), Bezout suspend quatre administrateurs sur cinq. Le président de l'administration municipale de Cosne traite Bezout de «proconsul aux vues perfides» (30). Finalement, le ministre Merlin de Douai va couvrir l'action de Bezout avec les attendus suivants :

«Les administrateurs départementaux sont destituables. Cette éventualité est prévue par la Constitution.

Il faut mettre les bons esprits en garde contre ces grands mots d'Élus du Peuple qu'ont sans cesse à la bouche les membres des différentes administrations qui ont été destitués depuis les dernières assemblées électorales. $\mathrm{La}$ Constitution veut indiquer par-là qu'il y a une immense différence entre l'élection des membres du Corps législatif et des tribunaux; et les membres des administrations départementales et municipales. Les premiers sont les véritables Élus du Peuple; ils ne peuvent être destitués que pour cause de forfaiture légalement jugée.

Les seconds, par la nature de leurs fonctions, ne sont que les agents du gouvernement, et leur élection, si l'on peut appeler ainsi les actes des assemblées électorales qui les concernent [...] ne sont de la part des électeurs qu'une

(29) A.D. Isère, Papiers Reverchon, 1 J 379,12 brumaire an IV - 3 novembre 1795.

(30) A.D. Nièvre, Registre de délibérations de l'administration départementale, fructidor an II-frimaire an IV - août 1794-décembre 1795. 
sorte d'indication donnée au gouvernement de leur civisme, de leur moralité, et de leurs talents. Le gouvernement peut donc les destituer [...]

Dans l'établissement d'une Constitution, il y a nécessairement un passage de l'ordre ancien à l'ordre nouveau, et ce passage n'est à proprement parler, ni constitutionnel, ni révolutionnaire, il est essentiellement mixte. Il y a donc toujours des lois transitoires; telle est la loi du 20 vendémiaire, relative aux pouvoirs des représentants du peuple en mission. » (31)

Merlin justifie l'action des envoyés, et pensent que, par leurs plaintes, les autorités destituées secondent les efforts des conspirateurs royalistes. Si on admettait que la loi du 20 vendémiaire est illégale, alors :

«Les opérations que le Directoire exécutif a faites en vertu de la loi du 3 brumaire le seraient aussi. C'est là que les protestataires veulent en venir. C'est en cela qu'ils secondent les efforts des royalistes pour ramener dans le sein de la Patrie les traîtres qu'elle réprouve, empoisonner par eux les autorités, tuer la Constitution par elle-même [...] » (32)

Le Directoire légalise par un message lu au Conseil des Cinq-Cents dans la séance du 28 brumaire an IV, la prolongation des pouvoirs accordés à ses commissaires extraordinaires :

«Le Directoire exécutif n'a pas cru voir dans la loi du 20 vendémiaire dernier l'obligation stricte de faire notifier aux divers représentants du peuple en mission son installation au moment même qu'elle serait effectuée. Il a pensé que cette loi avait au contraire pour objet de ne pas laisser tous les liens de l'ancien gouvernement se dissoudre avant que le nouveau ait eu le temps de former ceux qui sont créés par la Constitution ; aussi, lorsque la loi fut rendue, le rapporteur eut soin de faire remarquer que le Comité de salut public ne proposait pas de faire cesser les pouvoirs des représentants du peuple en mission dès le moment même où le Directoire serait installé et ferait connaître son installation à toute la République, mais seulement du jour où le Directoire exécutif notifierait spécialement son installation au représentant du peuple en personne : il ajouta que le Comité s'était déterminé à faire cette proposition par la considération des dangers qu'il y aurait à laisser le gouvernement sans agents près les armées ou dans certains départements [...] avant que les commissaires du pouvoir constitutionnel exécutif fussent nommés et en activité. » (33)

Toutes ces mesures sont justifiées par l'impératif du salut public. C'est ici que l'hypothèse des Compagnons de Jéhu joue pleinement son rôle ; peu importe la matérialité de son existence (34). La construction doit beaucoup aux rapports de Reverchon et de Fréron, repris et synthétisés à la tribune par Marie-Joseph Chénier (35).

(31) A.N., AF III 32, Papiers Merlin de Douai

(32) Ibid., À propos de la mission de Fréron à Marseille, 15 frimaire an IV.

(33) A. DEBIDOUR, Recueil des Actes du Directoire exécutif, t.I, p. 49.

(34) Bruno BENOIT, «Les compagnons de Jéhu ont-ils existé? », L'Histoire, 185,1995, pp. 16-18.

(35) Le Moniteur Universel, $\mathrm{n}^{\circ}$ du 9 messidor an IV (27 juin 1795). 
Dès le 6 messidor an IV (24 juin 1795), Chénier avait donné sa version des massacres commis au printemps à Lyon :

«Une association de scélérats ligués pour le meurtre s'est organisée à Lyon. Cette association mêlant les idées religieuses aux massacres, le cri du royalisme aux mots de justice et d'humanité, se fait appeler "Compagnie de Jésus". C'est elle qui répand dans cette commune une terreur nouvelle plus active encore et plus générale que celle qu'y répandaient Chalier et ses sanguinaires complices. C'est elle qui, sous prétexte de punir les atrocités commises par les brigands qui égorgeaient au nom du peuple, commet ellemême au nom du peuple, au nom de la Convention nationale, des atrocités plus révoltantes. C'est elle qui rappelle à grands cris les émigrés, qui protège leur rentrée sur le territoire de la République, qui les reçoit dans son sein, qui obéit à leurs vœux sacrilèges, qui réalise leurs espérances parricides, qui force l'asile domestique, et l'asile plus saint des prisons; qui montre publiquement, qui proclame ses listes de proscription; qui, les mains teintes de sang humain, vante hautement ses assassinats; qui dévoue aux poignards, non pas seulement les vrais terroristes que les tribunaux doivent punir, mais, sous le nom de terroristes, tous ceux qui, dans un poste public, ou dans leur vie particulière, ont servi la Révolution. C'est elle enfin, c'est cette compagnie exécrable qui chante sur les cadavres de ses victimes, et jouit en idée de la destruction prochaine de tous les patriotes, et du retour de la royauté, seul objet de ses désirs, seul but de ses complots, seule récompense de ses crimes. »

Chénier va reprendre et élargir cette argumentation, après les journées de vendémiaire qui semblent matérialiser l'existence d'un vaste complot contre-révolutionnaire. Un infléchissement significatif de la doctrine thermidorienne est apporté par Alexandre Villetard, au cours de la séance de la Convention du 29 vendémiaire an IV :

«Les assassinats commis sous Robespierre étaient aussi le crime des royalistes ; voilà comme on a avili les patriotes ; c'est ainsi qu'on les a traînés dans la boue, qu'on les a expulsés de toutes les places : c'est ainsi que le sort de la République a été mis dans les mains des royalistes [...] » (36)

Les hésitations sur la ligne politique, inhérentes à toute la période directoriale, sont présentes ici : une fraction des dirigeants thermidoriens substitue à la politique de la bascule, une politique de front républicain. Selon la politique de la bascule, le danger terroriste ou anarchiste, identifié comme tel, menace la stabilité de la République constitutionnelle, tout autant que le danger contre-révolutionnaire ou royaliste. Il y a donc trois courants politiques identifiés par les dirigeants; les royalistes, les anarchistes, les honnêtes gens ou patriotes sages, qui sont les seuls vrais partisans de la République. A contrario, selon la ligne de front républicain qui s'affirme entre vendémiaire et ventôse an IV, il n'y a qu'une unique conspiration royaliste aux vastes ramifications, et aux visages multiples (le brigandage, 
l'espionnage, la modération interprétée comme complicité avec l'ennemi, le trouble à l'ordre public) (37). Contre elle, pour défendre la République, sans trop s'arrêter à l'interprétation littérale du texte constitutionnel, il faut unir tous les vrais patriotes, y compris ceux qui furent un temps persécutés comme terroristes ou anarchistes. Il n'y a que deux partis, et pas de juste milieu : royalistes et républicains (38).

Dans cette perspective, il revient à Chénier, qui parle tout de suite après Villetard, de développer les multiples aspects de la conspiration royaliste, beaucoup plus vaste que la seule référence à la Compagnie de Jésus, mentionnée au début de l'été :

«Vous vous rappelez, représentants, que je vins, il y a trois mois, à cette tribune, vous dénoncer, au nom des Comités de salut public et de sûreté générale, les nombreux attentats commis dans la commune de Lyon. Les voiles dont quelques intrigants avaient enveloppé la vérité, sont déjà transparents.

C'est aux représentants du peuple qui sont dans le Midi, qu'il appartient de les soulever et de reconnaître tous les coupables.

Ils ne sont pas rassemblés dans une seule commune : dix départements, trente cités, ont vu se perpétuer ces scènes sanglantes.

À Marseille, à Tarascon, à Aix, dans le département des Bouches-duRhône ; à Avignon, à l'Isle, dans le département du Vaucluse ; à Nîmes, dans le département du Gard ; à Sisteron, dans le département des Basses-Alpes ; à Toulon, dans le département du Var; à Montélimar, dans le département de la Drôme, on a lutté de crimes avec les assassins de Lyon.

La fureur des royalistes ne s'est pas encore arrêtée dans ces communes, elle s'est promenée dans les contrées de l'Est; elle a souillé Saint-Étienne de Montbrison [sic] dans le département de la Loire; Bourg, dans le département de l'Ain ; Lons-le-Saulnier, dans le département du Jura. Elle a pénétré jusqu'à Sedan, au sein du département des Ardennes; dans le centre de la France, et à Rousières, district du Chinon [sic], département d'Indre-etLoire; et jusqu'aux portes de Paris, à la ferme du Bois-Blanc, commune d'Arainville, district de Corbeil, département de Seine-et-Oise... »

La théorie de la conspiration est le produit d'un double élargissement ; élargissement géographique, qui amalgame tout particulièrement les massacres du Midi et les massacres de la région lyonnaise, au sens large. La République de 1795 redevient la «citadelle assiégée » de 1793. L'autre amalgame constitutif de la théorie du complot réside dans la politisation du brigandage : une attaque de ferme, une malle-poste dévalisée, sont interpré-

(37) Bernard GAINOT, 1799, un nouveau jacobinisme ?, Paris, Éd. du CTHS, 2001, pp. 31-43 ; JeanRené SURATTEAU, «Les babouvistes, le péril rouge et le Directoire », dans Babeuf et les problèmes du babouvisme, Paris, Éditions sociales, 1963.

(38) Stephen CLAY, «Le massacre du fort Saint-Jean, un épisode de la terreur blanche à Marseille », dans Le tournant de l'an III (...), op. cit., pp. 569-583. 
tées comme des actions contre-révolutionnaires. Ainsi, se forge l'image du brigand royal, prototype de l'ennemi de l'intérieur. L'application de la loi républicaine, le contexte externe ayant une importance considérable que nous ne développerons pas dans le cadre de cette communication, est bien davantage une question d'ordre public (du domaine de la coercition), qu'une question d'espace public ( $\mathrm{du}$ domaine du consentement). Les personnalités influentes du régime directorial, même les plus libérales, ne diront pas autre chose (39).

En conclusion de son rapport, Chénier propose le projet de décret, qui est la véritable source des pouvoirs des futurs commissaires du gouvernement :

«Article $1^{\mathrm{er}}$ : Les maires et procureurs de communes qui n'ont pas dénoncé les auteurs et complices des assassinats commis dans plusieurs parties de la République, par les "compagnies de Jésus", les "compagnies du Soleil", et autres associations royalistes, sont destitués.

Article 2 : Les juges de paix et accusateurs publics qui n'ont pas poursuivi devant les tribunaux les auteurs et complices de ces massacres, sont pareillement destitués.

Article 3 : Les maires et procureurs de commune, juges de paix et accusateurs publics, qui se rendraient à l'avenir coupables d'une pareille négligence, seront punis de deux années de détention.

Article 4 : Les représentants du peuple en mission dans les départements, et le comité de législation, pour les départements où il n'y a pas de représentants du peuple en mission, sont chargés de destituer sans délai les autorités constituées qui sont dans le cas déterminé par les deux premiers articles. Ils sont chargés pareillement de faire traduire sur-le-champ devant les tribunaux, les auteurs et complices des assassinats mentionnés dans le présent décret, pour y être jugés conformément au Code pénal. »

C'est en vertu de ces larges pouvoirs d'épuration des autorités tout nouvellement constituées que Fréron est investi de sa mission dans le Midi. De même, Reverchon, investi des mêmes pouvoirs, ultérieurement conforté par l'aval de Merlin, peut enfin se déplacer vers Lyon dans l'hiver 1795-1796, où se place le point d'orgue de sa mission, avec l'installation d'un régime d'exception, qui sera la norme pendant toute la période directoriale dans la deuxième ville de France (40).

(39) Jean DE BRY, Le Moniteur universel, séance du Conseil des Cinq-Cents du 7 frimaire an VI (27 novembre 1797) : «... j'en ai la conviction morale ; ce n'est point s'amuser à combattre des chimères, ni ressasser ici des inculpations usées, que de dire que tous les désordres dont nous nous plaignons, dont les écrivains remplissent leurs feuilles, et dont tous les citoyens gémissent, sont l'effet du grand plan organisé par nos ennemis, exercé depuis le commencement de la Révolution, et dont le but est de rendre le régime républicain odieux, et le gouvernement représentatif impraticable... ».

(40)Bruno BENOIT, «Lyon et la République directoriale», dans La République directoriale, (dir. Philippe BOURDIN et Bernard GAINOT), T . 1, Clermont-Ferrand, Bibliothèque d'histoire révolutionnaire, 1998, pp. 261-276. 
La réhabilitation du personnel de l'an II est l'autre versant de ces missions «vendémiaristes». Reverchon retrouve un grand nombre de «patriotes» qu'il avait côtoyés en 1793, lors de la répression de la crise fédéraliste lyonnaise. Les liens sont particulièrement avérés dans le département de la Loire. Reverchon était déjà intervenu une première fois, pour faire libérer des suspects, en messidor an II ; à Commune d'Armes (Saint-Étienne), il s'agissait d'un conflit entre la Commission chargée pour le compte du gouvernement de la régie de la manufacture, et un groupe de révolutionnaires locaux, qui dirigeaient la Société populaire. Reverchon, appuyé par Noël Pointe, Claude Javogues, Boyer et Boisrond, avait fait libérer un certain nombre de suspects emprisonnés sur ordre de la Société populaire, dont l'ancien agent national Laforest (41). En brumaire an IV, ce sont désormais les «patriotes » désarmés lors de la «réaction » qu'il remet en selle; on retrouve dans ses Papiers une correspondance suivie avec plusieurs d'entre eux. Pendant tout le Directoire, il fait figure de «protecteur » auprès des autorités centrales et intervient en leur faveur, notamment à l'occasion des opérations électorales. Ces interventions se produisent très vite, dès son installation comme commissaire du gouvernement au début de l'an IV. Citons, à titre d'exemple, la lettre de Jean-François Michaud, capitaine dans la Garde nationale de Boën, qui dénonce le commissaire cantonal, homme de loi et notaire public. C'est un déserteur du régiment de Penthièvre-dragons, qui protège les assassins de la Compagnie de Jésus et du Soleil. Il a déserté en 1793, pour se mettre à la tête d'un groupe de rebelles lyonnais (42). Le passé proche refait surface, sous couvert cette fois de lutte contre la grande conspiration royaliste, dénoncée à Paris. Non, décidément, la Révolution n'est pas terminée...

Ce «moment inaugural» de la République directoriale peut être placé dans la perspective des remarques de Sergio Luzzatto dans L'autumna della rivoluzzionne :

«La proclamation de l'amnistie générale, conçue comme instrument de lutte contre la Contre-révolution, ressemble fort à une réhabilitation du personnel politique de l'an II. Dans ces conditions, le vote des conventionnels, le tout dernier jour de leur mandat, affaiblit à sa source le régime directorial, le vouant au destin ingrat de dépendre, alors même qu'il les rejette, des hommes compromis dans les événements de 1793. Le vote d'amnistie du 4 brumaire an IV contient en germe à la fois le coup d'État néo-jacobin [43] du 18 fructidor an V, et le coup d'État anti-jacobin du 22 floréal an VI. » (44)

(41) A.D. Isère, Papiers Reverchon, 1J378, Liste des personnes détenues par ordre du comité de surveillance de Commune d'Armes.

(42) Ibid., $1 \mathrm{~J} 379$.

(43) Formulation très discutable.

(44) Sergio LUZZATTO, L'automne de la Révolution. Luttes et cultures politiques dans la France thermidorienne, Paris, Honoré Champion, 2001, p. 325. 
Il faut cependant considérer que l'échec de la politique officielle du Directoire sur le plan local - la «pacification» des corps et des esprits tient aux difficultés à trouver un personnel d'exécution qui ne soit pas profondément impliqué dans les luttes civiles des années écoulées, avec son lot de cruautés et de vengeances. Le déficit des moyens excède naturellement la question du réinvestissement dans l'appareil d'État de l'ancien personnel «terroriste» ou «sans-culotte», pour rejoindre une autre remarque de Sergio Luzzatto :

«Tous les hommes politiques qui occupent le devant de la scène dans la République directoriale, de la droite à la gauche, à Paris et en province, disposent d'un excès de mémoire, sont affectés d'un déficit d'oubli qui les pousse à chercher sans cesse la revanche politique, si ce n'est la vengeance personnelle. Ceci explique les heurs et malheurs, entre 1795 et 1799, des institutions républicaines, trop chargées de souvenirs pour être perçues comme innocentes et vécues comme fondatrices. » (45)

En envisageant la transition brutale du régime révolutionnaire au régime constitutionnel, les conventionnels cherchent désespéramment à faire l'économie de cette indispensable politique de la mémoire (46). Mais l'aspect proprement institutionnel de la transition ne doit pas non plus être sousestimé. Si le discours d'assemblée met délibérément l'accent sur la séparation des pouvoirs, et si Montesquieu est invoqué plus souvent que Rousseau, il ne faut pas pour autant s'en tenir à la seule démarche théorique.

La pratique administrative reste marquée par la confusion des pouvoirs, et il semble bien que ce qui est mal maîtrisé en terme de théorie politique, ce soit cette notion de «gouvernement mixte», que l'on trouve pourtant chez Montesquieu, et au-delà cette incapacité à définir vraiment la place de l'Exécutif, entre subordination et arbitraire. Si les hommes politiques du Directoire ont eu la pratique des «situations mixtes », ils n'ont jamais pu dépasser les apories de la théorie constitutionnelle pour installer dans la durée le «gouvernement mixte».

Bernard GAINOT

Institut d'histoire de la Révolution française

Université Paris I Panthéon-Sorbonne

17, rue de la Sorbonne 75231 Paris Cedex 05 gainot@univ-parisl.fr

(45) $I d$, pp. 338-339.

(46) Jean-Clément MARTIN, « Histoire, mémoire, oubli ; pour un autre régime d'historicité », Revue d'histoire moderne et contemporaine, 47-4,2000, pp. 783-804. 


\section{Le « moment » vendémiaire an IV}

5 fructidor an III (22 août 1795) : la Convention adopte le nouveau texte constitutionnel.

20 fructidor an III (6 septembre 1795) : ouverture des assemblées primaires en province. Vote populaire de ratification de l'acte constitutionnel, puis de ratification du décret des deux-tiers.

10 vendémiaire an IV ( 2 octobre 1795) : loi sur la police intérieure des communes.

12 vendémiaire an IV (4 octobre 1795) : la Convention rapporte le décret du 21 germinal sur le désarmement des « terroristes » et celui du 5 ventôse qui a placé sous surveillance les fonctionnaires publics destitués après thermidor.

13 vendémiaire an IV (5 octobre 1795) : insurrection des sections parisiennes.

14 vendémiaire an IV (6 octobre 1795) : dénonciation d'une grande conspiration qui vise à pervertir les choix des assemblées primaires. Des «émissaires secrets» assuraient la correspondance entre les sections parisiennes et les centres de la conspiration à Lyon et dans le Midi.

20 vendémiaire an IV (12 octobre 1795) : ouverture des assemblées électorales.

À la suite d'un débat animé, la Convention adopte le décret du Comité

de sûreté générale qui accorde des pouvoirs étendus à des « représentants $\mathrm{du}$ peuple», «commissaires du gouvernement» (Fréron, Reverchon, Bezout, Goupilleau de Montaigu).

22 vendémiaire an IV (14 octobre 1795) : libération des « patriotes » emprisonnés à la suite des journées de prairial.

29 vendémiaire an IV (21 octobre 1795) : loi relative à la répression des menées de la conspiration contre-révolutionnaire (Compagnons de Jéhu et Compagnons du Soleil).

Les représentants en mission les plus « réacteurs » sont dénoncés pour complicité d'assassinats.

30 vendémiaire an IV (22 octobre 1795) : proposition d'un comité de cinq membres pour adopter des mesures de salut public.

Après un vif débat, le «front républicain » se fissure et la proposition est rejetée.

3 brumaire an IV (25 octobre 1795) : loi d'exclusion des prêtres réfractaires et des émigrés.

4 brumaire an IV (26 octobre 1795) : loi d'amnistie générale pour les faits se rapportant à la Révolution.

5 brumaire an IV (27 octobre 1795) : installation du Directoire exécutif. 